\title{
DIAGNÓSTICO DE UMA POPULAÇÃO \\ DA TERCEIRA IDADE
}

Marina Sousa Pinheiro Mota ${ }^{1}$

Carine Moura Ferreira²

Daniele Idalino Janebro ${ }^{3}$

Ione Ramos de Queiroz ${ }^{4}$

Maria do Socorro Ramos de Queiroz 5

resumo

Este estudo teve como objetivo traçar e avaliar o perfil das pessoas da terceira idade e desenvolver práticas educativas multidisciplinares que contribuam para melhorar a qualidade de vida. Tratou-se de uma pesquisa quantitativa realizada no município de Cabaceiras-PB e envolveu 41\% da população com faixa etária de 60 anos. Participaram 176 idosos, a faixa etária predominante foi de 60 a 69 anos ( $n=93$ ), o gênero feminino foi o mais freqüente, a maioria eram casados e moravam com a família e apenas 40 deles não tiveram oportunidade de estudar. Grande parte residia em casa própria e apenas 18 deles não estavam satisfeitos com o lugar onde moravam por falta de infra-estrutura da cidade; 98\% da amostra era aposentada $(n=172)$ e destes, 49 ainda exerciam alguma atividade, sendo a principal, do tipo laborativa. Dos participantes, 150 conseguiam desenvolver sozinhos suas atividades diárias, os que demonstraram insatisfação era devido a problemas de saúde. Com relação a perspectivas futuras, 130 revelaram ter ao menos algum projeto a ser realizado, no entanto, alguns deles estavam insatisfeitos com a velhice porque não conseguiam manter sua autonomia. Dos problemas de saúde, os mais frequentes foram hipertensão/cardiopatias, hipertensão/diabetes e doenças osteo-musculares. Mesmo apresentando certas limitações, 92\% ( $n=162)$ revelaram ser felizes por manter relacionamento familiar e social afetivo. Em alguns casos, a falta de apoio pode resultar numa ruptura do seu projeto existencial,

\footnotetext{
1 Discente do Curso de Farmácia da Universidade Estadual da Paraíba.

2 Discente do Curso de Farmácia da Universidade Estadual da Paraíba.

3 Farmacêutica Bioquímica. Doutora em Produtos Naturais, Discente das disciplinas Hematologia e microbiologia, Faculdade Maurício de Nassau.

4 Enfermeira do Programa Saúde da Família

5 Farmacêutica Bioquímica. Mestre em Ciência dos Alimentos, discente das disciplinas Deontologia e Legislação Farmacêutica e Estágio Supervisionado III do Departamento de Farmácia, Universidade Estadual da Paraíba. E-mail: queirozsocorroramos@yahoo.com.br
} 
originando a perda do significado da vida. O importante é viver com qualidade conseguindo manter a autonomia e o bem estar.

palavras-chave

Envelhecimento. Idoso. Terceira idade.

\section{Introdução}

O envelhecimento é universal e, por ser natural, não depende da vontade do indivíduo. Ou seja, todo ser nasce, desenvolve-se, cresce, envelhece e morre. Segundo Beauvoir (2003), a vida é dinâmica, em todo momento se perde o equilíbrio e mais adiante se o reconquista, é essa constante mudança que constitui a lei da vida. Assim, durante todo o percurso, a cada fase de desenvolvimento, ocorrem múltiplas transformações acompanhadas de próprios desafios.

Envelhecer também é considerado irreversível. Apesar de todo o avanço da medicina em relação às descobertas, aos tratamentos das doenças, às novidades farmacológicas, ao desenvolvimento de técnicas estéticas, nada é capaz de reverter tal processo,. Ele é heterogêneo e individual, em cada espécie há uma velocidade própria para envelhecer, variando de indivíduo para indivíduo e num mesmo indivíduo de órgão para órgão. O processo é ainda deletério, pois leva a uma perda progressiva das funções, o que dificulta a manutenção da homeostasia (DUARTE, 2008).

Carvalho et al. (2003) afirmaram que tal processo é um fenômeno complexo que inclui alterações fisiológicas e psicológicas relacionadas com as condições sociais vigentes. Dependendo do contexto (biológico, social, econômico e cronológico), o termo envelhecimento pode assumir significados diferentes. Do ponto de vista biológico, começa logo que nascemos, pois, trata-se de um processo contínuo ao longo do desenvolvimento humano; socialmente, as características pertinentes ao mesmo são percebidas de acordo com o contexto cultural e de geração para geração; economicamente, o que marca tal processo, em algumas sociedades, é a aposentadoria, definida em termos de saída do mercado de trabalho; e cronologicamente, dá-se a partir dos 60 anos, idade que corresponde à faixa etária da terceira idade.

Na velhice, tudo é atribuído à idade, são anos marcados por doenças, sequelas, declínio funcional, aumento da dependência, perda da autonomia, isolamento social e depressão, sem levar em conta as características da personalidade de cada indivíduo e do contexto socioeconômico em que está inserido. A desvalorização social da velhice e sua associação imediata à doença 
desestimulam muitas vezes o idoso a ter uma postura de maior investimento em sua saúde, como se nada pudesse repercutir positivamente ou amenizar limitações.

Para a Organização Mundial da Saúde (OMS), no ano de 2025, o Brasil estará incluído entre os 10 países do mundo com maior contingente de pessoas com 60 anos ou mais (WHO, 2008). Para Kalache (2005), o fato de a humanidade ter acrescentado 29 anos à sua expectativa de vida é a maior conquista do século XX e o grande desafio do século XXI. Não há dúvida de que o envelhecimento da população revela-se como uma tendência positiva, ligada intimamente à maior eficácia das medidas preventivas em saúde, à tecnologia dos tratamentos e dos fármacos, à uma melhor intervenção no meio ambiente e, sobretudo, à conscientização progressiva de que somos os principais agentes da nossa própria saúde.

Neste sentido, ressalta-se a importância do desenvolvimento de estudos que venham a analisar os aspectos econômicos, ambientais, bem como as transformações biológicas, psicológicas e sociais que envolvem o grupo da terceira idade. Feito isso, se pode então propor atividades educativas que promovam a saúde e a cidadania para que envelheça-se com autonomia e independência, desempenhando ainda atividades na sociedade.

Tendo em vista esses aspectos, o presente estudo teve como objetivo traçar e avaliar o perfil das pessoas da terceira idade e desenvolver práticas educativas multidisciplinares que contribuam para melhorar a qualidade de vida.

\section{Metodologia}

Foi realizado um estudo com abordagem quantitativa, descritiva e exploratória de corte transversal, tipo inquérito populacional domiciliar realizado em Cabaceiras-PB, no período de maio a outubro de 2008. O município dispõe de uma população de 4.281 habitantes, sendo que $10 \%$ correspondem à faixa etária acima de 60 anos. O critério de inclusão foi idade $\geq 60$ anos, totalizando uma amostra de $41 \%$ de idosos ( $n=176$ ). Os domicílios foram selecionados de maneira aleatória simples, por meio de amostragem por conglomerado, usando como unidade de referência o setor censitário urbano presente na secretaria de saúde.

Os dados foram coletados através de um questionário estruturado com perguntas abertas e fechadas. Os parâmetros avaliados para caracterizar as condições de vida dos idosos foram: faixa etária, gênero, estado civil, nível de escolaridade, relacionamento familiar, situação social, desempenho de ativi- 
dades diárias, situação previdenciária, tipo de moradia, problemas de saúde e perspectivas futuras.

O estudo foi realizado após aprovação do Comitê de Ética em Pesquisa da Universidade Estadual da Paraíba (UEPB) sob no 0389.0.000.133-06. Os participantes foram informados a cerca dos objetivos, da metodologia da pesquisa e após concordarem, assinaram o Termo de Consentimento Livre e Esclarecido.

A análise dos dados seguiu critérios da estatística descritiva e os resultados foram apresentados em forma de tabelas.

\section{Resultados}

Foram contatados 200 idosos, e destes, participaram da pesquisa 176; 16 se recusaram a responder ao questionário e 8 não responderam por serem portadores de problemas neurodegenerativos. A caracterização da amostra de idosos com relação às variáveis sócio-demográficas encontra-se na Tabela 1 .

A faixa etária predominante foi entre 60 e 69 anos, sendo o gênero feminino o mais frequente. Dos entrevistados, 97 (55\%) tinham vínculo conjugal e residiam com a família, formando núcleos pequenos, variando de dois a quatro habitantes.

No grupo pesquisado, 168 idosos (95\%) possuíam moradia própria e apenas 8 deles (5\%), estavam insatisfeitos com o lugar onde residem. Os principais motivos para a insatisfação foram a falta de pavimentação e o desejo de morar com os filhos em outro estado.

A escolaridade apresentou-se diversificada, cerca de 136 (77\%) da amostra frequentaram a escola e destas 78 (57\%) concluíram o Ensino Fundamental I. Com relação à situação previdenciária $(\mathrm{n}=173), 98 \%$ dos entrevistados eram aposentados e destes, 49 ainda exercem atividade remunerada, sendo as mais comuns: atividade mercantil, corte e costura, artesanato e agricultura.

A funcionalidade dos idosos foi avaliada a partir do seu desempenho referido nas atividades cotidianas realizadas em sua residência. Observou-se que 150 idosos (85\%) tinham condições de realizar as atividades diárias com relação ao seu autocuidado (vestir-se, movimentar-se, higienizar-se e alimentar-se). No entanto, 24 idosos (13\%) declararam-se insatisfeitos por realizarem tais atividades com dificuldades, devido a problemas de saúde. Apesar da idade avançada, verificou-se que 130 idosos (73\%) estavam satisfeitos com a velhice e acreditavam em melhores expectativas de vida e 46 deles (35\%) não apresentaram esperança de viver. 
Quanto aos problemas de saúde, apenas $12 \%$ dos entrevistados ( $\mathrm{n}=21)$ não os apresentaram. Dentre os que se queixaram de problemas de saúde ( $\mathrm{n}=155)$, a hipertensão/cardiopatias e as doenças osteo-musculares foram os mais citados. Dentre os grupos farmacológicos mais utilizados pela população estudada, foi observado que $56 \%(n=88)$ referiram-se aos anti-hipertensivos/cardiovasculares, seguido por analgésicos e antiinflamatórios com $18 \%$ $(\mathrm{n}=32)$. Apesar de alguns problemas de saúde, afetivos, financeiros e incapacidade física pertinente a esta fase da vida, 92\% (n=162) dos idosos revelaram ser muito felizes.

\section{Discussão dos Resultados}

De acordo com o resultado obtido, o gênero feminino foi o mais frequente, estando de acordo com a literatura consultada. A velhice é um "território feminino" devido à maior longevidade desse gênero, como também a solidão que, nos anos tardios, é uma vivência feminina, em grande parte devido à viuvez. Já com os homens, quando se dá tal ocorrência, eles permanecem sozinhos por pouco tempo, geralmente se casando com mulheres mais jovens. A predominância feminina também ocorre porque as mulheres têm maior expectativa de vida, são mais atentas ao aparecimento de sintomas, têm um conhecimento maior das doenças e utilizam mais os serviços de saúde do que os homens (TRAVASSOS et al., 2002).

Dos entrevistados, 97 tinham vínculo conjugal. Para Ramos (2002), o casamento e a paternidade são variáveis que tem um grande efeito em comportamentos (consumir álcool, fumar ou ter hábitos de vida desordenados) que comprometem a saúde. Porém, não basta apenas a presença física, é necessário o apoio, especialmente para aqueles que apresentam dependência no seu dia-a-dia.

Quanto à moradia, eles revelaram possuir imóvel próprio e gostar da cidade onde residem. Para Anderson (2008), ter moradia própria constitui um dos aspectos centrais da condição de vida e de bem-estar dos idosos. A satisfação com o local onde moram é um fator importante na qualidade de vida deles, visto que representa sua integração com a comunidade, com a segurança e garantia de assistência médica e social.

Muitos participantes frequentaram a escola, sendo o Ensino Fundamental I o nível mais obtido. Ross e Ling Wu (1996) e Ramos (2002) observaram em seus estudos a relação entre educação e as vantagens cumulativas no status da saúde nas diferentes faixas etárias. Eles associaram níveis educacionais à promoção da boa saúde em idades mais avançadas, pois a educação 
é uma variável capaz de protelar problemas de saúde, no sentido de que ela permite uma acumulação de vantagens.

Apesar dos entrevistados, em sua maioria, serem beneficiados pelo Instituto Nacional do Seguro Social (INSS), alguns ainda exercem atividade remunerada ou não. $\mathrm{O}$ trabalho para idosos é um tópico que merece ser analisado com cuidado. Se por um lado ele pode ser um indicador da capacidade de manter atividade laborativa, por outro, ele pode refletir a necessidade de continuar trabalhando, para garantir a sobrevivência (ANDERSON, 2008).

Foi observado que a maior parte dos idosos tem condição de realizar atividades diárias e está satisfeita com a sua auto-percepção. Correlacionando esses dados com a saúde, o idoso, de uma maneira geral, tem maior propensão a conviver com problemas de saúde, o que não necessariamente o incapacita fisicamente e emocionalmente a realizar as atividades rotineiras (ARAÚJO; ALVES, 2008).

Segundo Silva, Fossati e Portella (2007), Silveira e Faro (2008) e Veras (2007) é satisfatório para o idoso sentir-se útil e satisfazer-se com o que faz. Para isso é importante auxiliá-lo na reorganização do seu tempo e na busca alternativa para realização pessoal no lazer e sociabilização. Quanto maior o senso de auto-eficácia, mais intensos são os sentimentos de satisfação.

Avaliando a presente amostra verificou-se que muitos estão satisfeitos com a velhice e acreditam em melhores perspectivas de vida. Ramos (2002) também realizou um estudo semelhante em São Paulo e observou que a grande maioria dos idosos revelou dispor de pelo menos um projeto para a vida. Aqueles que não têm mais esperança de viver, caem numa situação de anomia, de depressão, de desinteresse na vida, que é dramática e aproxima de situações de dependência.

Os idosos que consideram a velhice como um fenômeno natural, dão mais sentido à vida, participam mais do seu meio e da sociedade, sendo felizes. Eles correspondem, portanto, ao auto-reconhecimento de aspectos positivos, como um sistema de valores estável, como por exemplo, sensatez, amadurecimento e outros e de determinadas vantagens como a diminuição da responsabilidade e do trabalho, aumento do tempo livre para o lazer e a auto-estima elevada (RIBEIRINHO, 2008).

Os problemas de saúde presentes na população estudada corroboram com outros estudos citados na literatura. Martins (2008) investigou que nessa faixa etária os problemas mais frequentes são as Doenças Crônicas Não Transmissíveis (DCNT), como Hipertensão Arterial e Diabetes Mellitus e as doenças osteo-musculares. A pesquisadora acrescentou que em muitos casos 
é difícil distinguir se a causa é decorrente do processo de envelhecimento ou se são manifestações patológicas.

A velhice é um período da vida com uma alta prevalência de DCNT, limitações físicas, perdas cognitivas, sintomas depressivos, declínio sensorial, acidentes e isolamento social. Entretanto, tem crescido o interesse em estabelecer quais os fatores que isoladamente ou conjuntamente, melhor explicam o risco que um idoso tem de morrer em curto prazo (ANDERSON, 2008).

De acordo com Ramos (2002), pesquisas têm mostrado que a ligação entre status socioeconômico e saúde, baixo nível de educação e de renda está associada com restrito acesso ao suporte social e, consequentemente, com altas taxas de morbidade e mortalidade.

O suporte social pode exercer um papel essencial promovendo e mantendo a saúde física e mental. As pessoas nas classes sociais mais baixas são mais isoladas e recebem menos assistência do que aquelas de classes mais altas (RAMOS, 2002). Para o mesmo autor, as pessoas em posição inferior na escala socioeconômica estão mais propensas a desenvolver problemas de saúde, pois estão mais expostas aos riscos físicos, químicos, biológicos e psicológicos que afetam a saúde, do que os mais favorecidos.

Foi observado que, entre os grupos farmacológicos mais utilizados, estavam os anti-hipertensivos/cardiovasculares e os analgésicos e antiinflamatórios. Os analgésicos narcóticos exibem alterações variáveis de sua farmacocinética em relação à idade do usuário. Os pacientes idosos são mais sensíveis aos efeitos respiratórios desses agentes, por isso este grupo de fármacos deve ser utilizado com cautela até que se estabeleça a sensibilidade particular de cada indivíduo. Com relação aos antiinflamatórios não-esteroidais (AINES), até $60 \%$ dos idosos pode desenvolver ulceração péptica ou hemorragia assintomaticamente e, apesar deste risco, esses medicamentos estão entre os mais prescritos para a população geriátrica (KATZUNG, 2008).Os medicamentos psicotrópicos (sedativos, antidepressivos e tranquilizantes) se destacaram pelo abuso e pela dependência entre os idosos, o que pode causar insônia, confusão mental e favorecer acidentes provocados por tonturas (AZEVEDO, 2008).

Entre os entrevistados a maioria revelou ser feliz. Segundo Alcorta, (2008), o caminhar da felicidade está num meio termo chamado "amadurecimento" que é inerente ao idoso e este é justamente sua imensa vantagem sobre todas as outras idades. Depois de certo tempo não se pode tudo; mas tudo que é possível é muito melhor do que aquele tudo que acreditamos estar sempre ao nosso lado, mas nunca chegamos a alcançar. 
Paschoal (2008), Freire Junior e Tavares (2008) elegeram a felicidade como o principal fator a ser identificado com qualidade de vida, pois ela é o mais geral dos conceitos de bem estar, ligando-se de forma muito íntima às vontades e aos objetivos das pessoas. Desta maneira, alguém é feliz porque seus desejos e objetivos são ou estão sendo realizados. Como eles tendem a ser múltiplos, são feitas escolhas, e esse sentimento floresce quando o objetivo julgado prioritário foi preenchido. A partir dessas constatações pode ser compreendido que uma boa convivência, a autonomia preservada e a saúde são essenciais para manter o bem estar. Assim, seria como se esses objetivos superassem todos os outros problemas e isso os preenchessem com um sentimento de felicidade.

\section{Conclusão}

Conhecer o perfil de uma população idosa é importante para apresentar aos gestores um diagnóstico e os indicadores básicos, tais como idade, gênero, nível educacional, condições socioeconômicas e a descrição dos problemas e necessidades que afetam a população-alvo, com especial ênfase naqueles que influenciam o estado de saúde e o bem-estar geral. Através das informações obtidas, será possível traçar políticas públicas voltadas para esse grupo etário que contribuirão para uma longevidade mais saudável e promissora em termos de realizações de projetos e sonhos adiados.

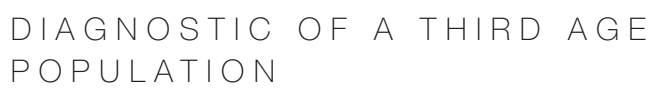

This study aimed to evaluate and draw up the elderly people profile and develop multidisciplinary educational practices that improve their life quality. It was a quantitative research conducted in CabaceirasPB town which involved $41 \%$ of the population aged about 60 years old. 176 elderly took part in the research, the predominant age group was 60 to 69 years old ( $n=93$ ), female ones were the most frequent, most of the interviewed were married and lived with their family and only 40 of them had no opportunity of studying. Many of the elderly live in their own home and only 18 of them were not satisfied with the place where they lived because of the town lack of infrastructure; 98\% of the sample was retired $(n=172)$ and 49 of them still were not carrying out any activity; the most common one 
was the laborious. 150 of the participants could develop their own

daily activities by themselves; the ones, who showed discontentment, did so, due to health problems. Regarding future prospects, 130 have revealed at least one project to carry out, however, some of them were dissatisfied with their aging because they could not keep their own autonomy. The most common health problems were hypertension/heart disease, hypertension/diabetes and osteo-muscular diseases. Even having some limitations, 92\% ( $n=162)$ of the elderly proved to be happy by maintaining affective family and social relations. In some cases a lack of support may result in the ending of the elderly existential project, causing the loss of the life meaning. The important thing is to have a good life quality, managing to keep independency and well-being.

keywords

Aging. Elderly. Third age.

\section{referências}

ALCORTA, Arturo. Escola da bicicleta. Idosos e a bicicleta. Disponível em http://www. escoladebicicleta.com.br/ Obtido em 03/06/2008.

ANDERSON, Maria Inez Padula. Saúde e condições de vida do idoso no Brasil. Textos sobre Envelhecimento, Rio de Janeiro, v. 1, n. 1, 1998. Disponível em unw.unati.uerj. br/tse/scielo.php?script=sci_abstract\&pid=S1517-59281998000100002\&lng=pt\&nrm =iso. Obtido em: 10/01/2008.

ARAÚJO, Tereza Cristina Nascimento; ALVES, Maria Isabel Coelho. Perfil da população idosa no Brasil. Textos sobre Envelhecimento, Rio de Janeiro, v.3 n.3, fev. 2000. Disponível em: http://www.cuidardeidosos.com.br/wp-content/uploads/2008/05/NeIhice\%20numa\%20perspectiva\%20de\%20futuro\%20saud\%C3\%A1 vel.pdf\#page=98 Obtido em: 10/01/2008.

AZEVEDO, João Roberto de. A utilização de medicamentos na terceira idade. Disponíve em: wnw.saudevidaonline.com.br/artigo102.htm. Obtido em: 08/06/2010.

BEAUVOIR, Simone de. A velhice. 5. ed. Rio de Janeiro, Nova Fronteira, 2003, p. 712.

CARVALHO, Ediana Nogueira de; SILVA, Francisca Rosângela da; MELO, Martha Teresa Siqueira Marques; CARVALHO, Cecilia Maria Resende Gonçalves de. Avaliação da qualidade nutricional das refeições servidas aos idosos em instituição asilar. Revista Estudos Interdisciplinares sobre o Envelhecimento, Porto Alegre, v. 5, p. 119-136, 2003.

DUARTE, Luzia Travassos. Envelhecimento: processo biopsicossocial. Trabalho Monográfico para o Curso Virtual "Educación para el Envejecimento". Disponível em: http:// www.psiconet.com/tiempo/monografias/brasil.htm. Obtido em: 10/01/2008.

FREIRE JÚNIOR, Renato Campos; TAVARES, Maria de Fátima Lobato. A saúde sob o olhar do idoso institucionalizado: conhecendo e valorizando sua opinião. Interface: comunicação, saúde, educação, Botucatu, v.9, n.16, p.147-58, set. 2004/fev. 2005. Disponível em: http://mww.scielo.br/pdf/icse/v9n16/v9n16a12.pdf. Obtido em 08/06/2008

KALACHE, Alexandre. Um mundo mais velho. Revista Veja. São Paulo, ed.1912, a. 38, n. 27, p. 11-15, jul/2005. 

Farmacologia Básica e Clínica. 5.ed. Rio de Janeiro, p. 899-906, 2003.

MARTINS, Rosa Maria Lopes. Envelhecimento e saúde: um problema social emergente. Millenium (revista do Instituto Superior Politecnico de Viseu). Viseu, Portugal, n.27, abril 2003. Disponível em: http://www.ipv.pt/millenium/Millenium27/14.htm. Obtido em: 21/03/2008.

PASCHOAL, Sérgio Márcio Pacheco. Qualidade de vida do idoso: elaboração de um instrumento que privilegia sua opinião. 2000. 263f. Dissertação (Mestrado em Medicina) São Paulo: Faculdade de Medicina, Universidade de São Paulo, 2001.

RAMOS, Marilia P. Apoio social e saúde entre idosos. Sociologias, Porto Alegre, a. 4, n. 7. p. 156-175, jan/jun, 2002.

RIBEIRINHO, Carla. Projeto de Vida: A vida continua a ter sentido. Disponível em: http:// unw. solidariedade.pt/sartigo/index.php?x=1313. Obtido em: Obtido em: 22/03/2008.

ROSS, Catherine E.; LING-WU, Chia. Education, age and the cumulative advantages in health. In: Joumal of Heatth and Social Behavior, New York, v. 37, n.1, 1996, p.104-120.

SILVA, Carine Alves da; FOSSATTI, Anderlei Fabiano; PORTELLA, Marilene Rodrigues. Percepção do homem idoso em relação às transformações decorrentes do processo do envelhecimento humano. Revista Estudos Interdisciplinares sobre o Envelhecimento, Porto Alegre, v. 12, p. 111-126, 2007.

SILVEIRA, Solange Convento; FARO, Ana Cristina Mancussi e. Contribuição da reabilitação na saúde e na qualidade de vida do idoso no Brasil: reflexões para a assistência multidisciplinar. Revista Estudos Interdisciplinares sobre o Envelhecimento, Porto Alegre, v. 13, n.1, p. 55-62, 2008

TRAVASSOS, Cláudia; VIACAVA, Francisco; PINHEIRO, Rejane; BRITO, Alexandre. Utilização dos serviços de saúde no Brasil: gênero, características familiares e condição social. Revista Panamericana de Salud Publica / Pan American Journal of Public Health, v. 11, n. 5/6, p. 365-373, 2002.

VERAS, Renato. Fórum: envelhecimento populacional e as informações de saúde do PNAD: demandas e desafios contemporâneos. Cademos de Saúde Pública, Rio de Janeiro, v. 23, n. 10, p. 2463-2466, out. 2007.

WHO, WORLD HEALTH ORGANIZATION. The uses of Epidemiology in the study of the elderly. Geneva. WHO. 1984, Technical Report Series 706. Disponível em: http://whallibdoc.who.int/trs/WHO_TRS_706.pdf. Obtido em: 11/01/2008.

Recebido: 14-03-2009

$1^{\text {a }}$ Revisão: 30-06-2009

$2^{a}$ Revisão: 17-05-2010

Aceite Final: 18-05-2010 